\title{
Green Method, Optical and Structural Characterization of ZnO Nanoparticles Synthesized Using Leaves Extract of M. oleifera
}

\author{
José Vulfrano González-Fernández ${ }^{1, *}$, Diego David Pinzón-Moreno ${ }^{1}$, Antony \\ Alexander Neciosup-Puican ${ }^{2}$ and María Verónica Carranza-Oropeza ${ }^{1}$ \\ ${ }^{1}$ Facultad de Química e Ingeniería Química, Universidad Nacional Mayor de San Marcos, Lima, 15081, Perú \\ ${ }^{2}$ Centro de Investigaciones Tecnológicas, Biomédicas y Medioambientales, Universidad Nacional Mayor de San Marcos, Lima, \\ 15081, Perú \\ *Corresponding Author: José Vulfrano González-Fernández. Email: fanogf@gmail.com
}

Received: 08 May 2021 Accepted: 21 June 2021

\begin{abstract}
$\mathrm{ZnO}$ nanoparticles (ZnO-NP) present innovative optical, electrical, and magnetic properties that depend on specific characteristics, e.g., size, distribution, and morphology. Thus, these properties are essential to address various applications in areas such as electronics, medicine, energy, and others. In addition, the performance of this $\mathrm{ZnO}$ NP depends of their preparation which can be done by chemical, physical, and biological methods. Meanwhile, nowadays, the main interest in developing $\mathrm{ZnO}-\mathrm{NP}$ synthesis through biological methods bases on the decrease of use of toxic chemicals or energy applied to the procedures, making the process more cost-effective and environmentally friendly. However, the large-scale production of nanoparticles by green synthesis remains a big challenge due to the complexity of the biological extracts used in chemical reactions. That being the case, the preparation of $\mathrm{ZnO}-\mathrm{NP}$ using Moringa oleifera extract as an alternative biological agent for capping and reduction in synthesis was evaluated in this work. Then, the results based on the analysis of the optical and structural characterization of the ZnO-NP obtained by employing UV-Vis, DLS, zeta potential, XRD, ATR-FTIR, and FE-SEM indicate mostly the presence of spherical nanosized material with a mean hydrodynamic diameter of $47.2 \mathrm{~nm}$ measured by DLS and a mean size diameter of $25 \mathrm{~nm}$ observed with FE-SEM technique. Furthermore, in FE-SEM images a homogeneous dispersion and distribution is observed in the absence of agglutination, agglomeration, or generation of significant lumps of the $\mathrm{ZnO}-\mathrm{NP}$. The XRD analysis showed that heat annealing induced the crystallite size favoring their monocrystallinity. Those obtained data confirm the synthesis of $\mathrm{ZnO}-\mathrm{NP}$ and the absence of impurities associated with organic compounds in the annealed samples. Finally, those results and low-cost production present to the synthesized $\mathrm{ZnO}-\mathrm{NP}$ by this biological method as a useful material in several applications.
\end{abstract}

\section{KEYWORDS}

$\mathrm{ZnO}$ nanoparticles; green chemistry; Moringa oleifera extract; optical characterization; structural characterization

\section{Introduction}

In recent years, the $\mathrm{ZnO}$ compound has been widely studied and it is usually described as a strategic, functional, versatile, and promising inorganic material with a broad range of applications [1,2]. $\mathrm{ZnO}$ is an n-type semiconductor member of the II-VI family characterized by a direct bandgap in the near UV 
spectrum at room temperature $(\sim 3.3 \mathrm{eV})$. $\mathrm{ZnO}$ crystallizes in the wurtzite, zinc blende, and rock-salt structures depending on the synthesis method, precursors, and experimental conditions [3,4]. This material also exhibits a high exciton binding energy of $60 \mathrm{meV}$ compared with the thermal energy $(26 \mathrm{meV})$ at room temperature. Also, it has intrinsic and unique properties such as stability at high temperatures, piezoelectricity, conductivity, a broad range of radiation absorption, chemical compatibility, durability, among others [5,6].

In general, nanoparticles exhibit new or improved properties that are supported by specific characteristics such as size, distribution, and morphology [7]. Furthermore, unlike their bulk counterparts, nanoparticles have a reduced size associated with high surface/volume ratio values that increase as the size of the nanoparticles decreases, making the particles highly reactive with prominent physical properties [8]. By combining $\mathrm{ZnO}$ material with the low dimensional scale, new properties of $\mathrm{ZnO}$ nanoparticles (ZnO-NP) are achieved. Several applications have been discovered or innovated over decades in different areas as renewable energy [9], biomedicine [10], sensing [11], construction and food industry [12,13], electronics [14], pharmaceutical [15], environmental sustainability [16], materials [17], agriculture [18], among many others.

Nanoparticles can be obtained using different techniques based on chemical (coprecipitation, sol-gel, chemical reduction, electrochemical, microemulsion, pyrolysis, photochemical, sonochemical, solvothermal, among others) [19-21], physical (milling, arc discharge, electron beam lithography, spray pyrolysis, ion implantation, laser ablation, inert gas condensation, mechanical grinding, vapor-phase plasma, ultrasonic irradiation, among others) [22-24] or biological (using extracts of plants, algae, microorganisms, enzymes, biomolecules or agro-industrial wastes, among others) $[7,25,26]$ methods.

For chemical synthesis, a common method to obtain $\mathrm{ZnO}-\mathrm{NP}$ is coprecipitation. By this method, it is possible to obtain a wide range of nanoparticle diameters and morphologies. Coprecipitation is considered an easy, cost-effective, and convenient technique because it does not require complex process parameters, any expensive and sophisticated equipment, or any large space area for the set-up [27]. Semiconductor nanoparticles, based on $\mathrm{ZnO}$ material, obtained by this method are usually applied in technology due to their high crystallinity and optical properties. In contrast, some chemical materials related to this technique are usually non-eco-friendly compounds, also the cost of these high-quality nanoparticles could be unnecessary for 'simple' applications, for example, in agriculture. In this case, the effective surface area of nanosized material is the desired property; the nanoparticle crystallinity has not a big influence when it is used as nanofertilizer, for instance [28].

In the case of physical methods, the mechanochemical synthesis or milling is frequently used to produce nanomaterials. In this method, mechanical energy is used to grind down powders by colliding balls with the powder due to the rapid rotation of the mill. High-energy ball milling is used over bulk powders to obtain smaller and more uniform sizes, even at the nanoscale [29]. During this procedure, the mechanochemical process occurs during repeated collisions, whereby mechanical energy is used to activate chemical reactions, which means it is a solvent-free synthesis method. ZnO-NP are also obtained by this method obtaining a wide range of nanoparticle diameters and good crystallinity but limited morphologies [30]. Despite the fact that it is a solvent-free synthesis, the mechanical process and sometimes the temperature annealing demand a high energy consumption making it not eco-friendly enough.

On the other hand, biological synthesis is considered an eco-friendly process to obtain ZnO-NP, which has arisen as a green alternative for the synthesis of nanomaterials. While chemical and physical methods may lead to high energy consumption a high temperature or pressure is required in the process [31,32].

The main interest in developing $\mathrm{ZnO}-\mathrm{NP}$ synthesis through biological methods has its basis on the low (or null) use of toxic chemicals and/or energy applied to the procedures, which makes the process more costeffective and environmentally friendly. Plants (leaves, flowers, seeds, peels, or roots) are the most important 
and strategic resource for the $\mathrm{ZnO}-\mathrm{NP}$ biosynthesis because other bioresources as fungi or microbes require sterilization processes or controlled conditions for their cultivation. The importance of this method, by using plant-based extracts to produce metal and oxide nanoparticles has been widely reported [33-35].

For biological synthesis, the natural extracted aqueous solution contains essential phytochemical compounds for the formation of the particles at nanoscale acting as capping and reducing agents transforming metal ion to metal oxides. Depending on the part of the plant, different functional groups as flavonoids, vitamins, polysaccharides, amino acids, alkaloids, among others, appear in the aqueous extract that, being used as the reaction solvent, significantly alters the chemical dynamics of the synthesis and, consequently, the type of nanoparticles as a product. By using extract leaves, in the particular case of the Moringa oleifera, $\mathrm{ZnO}-\mathrm{NP}$ with spherical symmetry is obtained by this method. This plant has, mainly, flavonoids (quercetin, kaempferol, myricetin), vitamins (L-ascorbic acid, retinol, niacin), glucosides (flavanol glucoside, benzoic acid glucosides, benzaldehyde 4-O- $\beta$-glucoside), phenolic acid (ellagic acid, gallic acid, chlorogenic acid, caffeic acid) [36-39], being flavonoids and phenolic acids the most important compounds of Moringa oleifera in the role of the reduction process to synthesize ZnO-NP. Since organic compounds are used in the process, the temperature treatments of leaves, drying and extracting processes, result as an important factor to control.

The large-scale production of nanoparticles by green synthesis remains a big challenge due to the complexity of the biological extracts used in chemical reactions. The mechanisms of formation during the nanoparticle synthesis is still be an important topic to be elucidated when they are produced at industrial quantities $[40,41]$.

In this work, the methodology for obtaining ZnO-NP using Moringa oleifera extract as an alternative agent for capping and reduction in synthesis is presented. Additionally, the analysis of the optical and structural characterization of the ZnO-NP obtained employing the ultraviolet-visible absorption spectroscopy (UV-Vis), dynamic light scattering (DLS), zeta potential, X-ray diffraction spectroscopy (XRD), Fourier transform infrared spectroscopy (ATR-FTIR), and field emission scanning electron microscopy (FE-SEM) are presented.

\section{Materials and Methods}

\subsection{Preparation of the Extracts}

The leaves of Moringa oleifera were produced by local farmers in the central and north coast from Peru and they were acquired in a small traditional market in Lima. Dry leaves of the Moringa oleifera plant were washed several times with tap water, rinsed with distilled water, and dried at $40^{\circ} \mathrm{C}$ in an air-fluxed oven to remove moisture.

The extract used for the reduction of zinc ions $\left(\mathrm{Zn}^{2+}\right)$ to $\mathrm{ZnO}-\mathrm{NP}$ was prepared by placing $13 \mathrm{~g}$ of fine cut leaves in $500 \mathrm{~mL}$ glass beaker along with $400 \mathrm{~mL}$ of ultrapure water $(18.2 \mathrm{M} \Omega \cdot \mathrm{cm})$ at $50^{\circ} \mathrm{C}$ by $2 \mathrm{~h}$ under magnetic stirring $(500 \mathrm{rpm})$, and a light-yellow aqueous solution was obtained. The extract was let to cool at room temperature and to be vacuum-assisted filtered by using a medium flow Whatman filter paper No. 1 (11 $\mu \mathrm{m}$ pore diameter) to avoid organic solid remains. The extract was stored in a refrigerator to be used for further experiments.

\subsection{Green Synthesis of ZnO Nanoparticles}

For the $\mathrm{ZnO}$-NP synthesis (summarized in Fig. 1), $100 \mathrm{~mL}$ of Moringa oleifera leaves extract was taken in a $250 \mathrm{~mL}$ glass beaker and heated to $70^{\circ} \mathrm{C}$ using a magnetic stirrer-heater at $500 \mathrm{rpm}$. When extract temperature reached $70^{\circ} \mathrm{C}, 3.3 \mathrm{~g}$ of $\mathrm{Zn}\left(\mathrm{NO}_{3}\right)_{2} \cdot 6 \mathrm{H}_{2} \mathrm{O}$ (zinc nitrate hexahydrate, purum p.a., purchased from Sigma Aldrich) was added as the precursor. The $\mathrm{pH}$ of the mixture was monitored at the beginning of the reaction with a Hanna Instruments model Edge HI2020 pH-meter, 4.5 was obtained. This mixture 
was heated and stirred for $2 \mathrm{~h}$. A yellow precipitation appeared at the bottom of the beaker, which was collected and heated at $70^{\circ} \mathrm{C}$ in an oven until completely dry. A light brown colored paste was obtained, and this was collected, grinded, and crushed with a mortar and pestle. Then, this powder was placed in an air muffle furnace at $600^{\circ} \mathrm{C}$ for $2 \mathrm{~h}$ of annealing to obtain white powder $\mathrm{ZnO}-\mathrm{NP}$. The sample was also mashed in a mortar-pestle and sieved to get a finer nature for characterization by using different techniques.

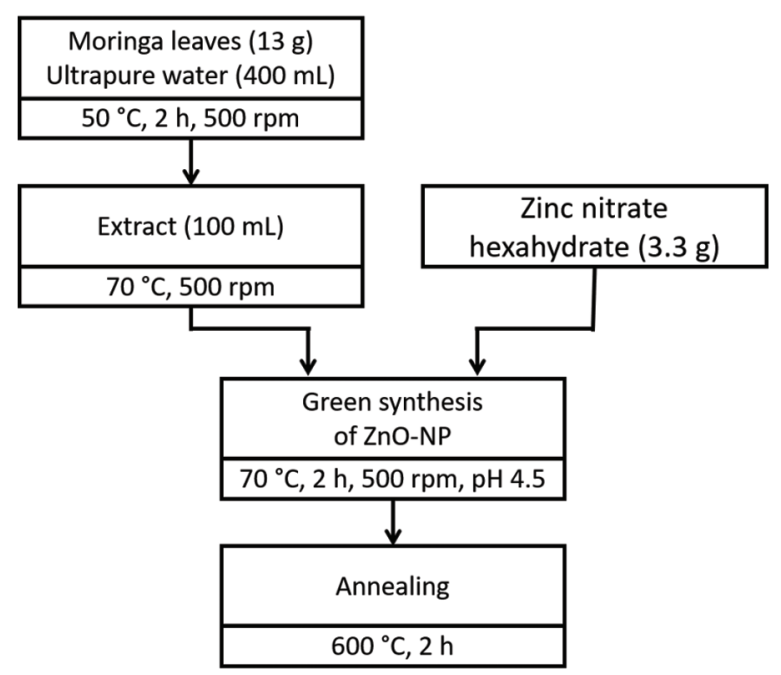

Figure 1: Summarized flowchart of green synthesis $\mathrm{ZnO}$ nanoparticles

\subsection{Characterization of ZnO Nanoparticles}

\subsubsection{Ultraviolet-Visible Absorption Spectroscopy (UV-Vis)}

The green synthesized ZnO-NP were confirmed and optically characterized by using a UV-Visible spectrophotometer (Libra S22, Biochrom, Ltd., Cambridge, England). Synthesized nanopowders were dispersed in $5 \mathrm{ml}$ of ultrapure water $(18.2 \mathrm{M} \Omega \cdot \mathrm{cm})$ and they were sonicated for $10 \mathrm{~min}$ at room temperature to guarantee a powder dispersion in the solution. The absorption spectra were recorded in the wavelength range of $200-800 \mathrm{~nm}$ (Step $1 \mathrm{~nm}$ ). The UV-Vis characterization gives us the insight on the actual formation of nanoparticles by a surface plasmon resonance effect.

The bandgap energy of semiconductors (including nanosized materials as films or structured particles) can be estimated by different methods and approximations. In this work we are using the Tauc relation [42] to study the optical bandgap of ZnO-NP:

$$
\alpha h v=B\left(h v-E_{g}\right)^{n}
$$

where $h v$ is the proton energy, $B$ is a proportionality constant, $E_{g}$ is the bandgap energy of the material, $n$ represents the power factor of the transition mode which is dependent upon the nature of the material, whether it is crystalline or amorphous. The absorption coefficient $\alpha$ has an important role in the Tauc plot method, it depends on the absorbance line shape $A$ and the thickness $t$ of the sample as the following fundamental relation:

$\alpha=2.3026 \mathrm{~A} / \mathrm{t}$ 


\subsubsection{Dynamic Light Scattering (DLS) and Zeta Potential}

The average size and dispersion of ZnO-NP were evaluated through dynamic light scattering (also called photon correlation spectroscopy or PCS) measured with a NICOMP Nano Z3000 System, which provides extreme versatility and high sensitivity. DLS is a widely used technique for the determination of particle size in an aqueous medium. The light scattered by nanoparticles in suspension will fluctuate with time and can be related to the particle diameter [43]. Measurement was done using a solid-state $\mathrm{He}-\mathrm{Ne}$ laser at a wavelength of $632.8 \mathrm{~nm}$ with a $4.0 \mathrm{~mW}$ at $23^{\circ} \mathrm{C}$.

Average diameters were obtained with the software provided by NICOMP (ZPW 388-V2.18). The light scattering from the Brownian-motion of the $\mathrm{ZnO}$ nanoparticles causes photon count fluctuations on the detector. Then, a correlation function is created to determine the translation diffusion coefficient $D$. The Stokes-Einstein equation is used to calculate the spherical particles of radius $R$ :

$$
D=\frac{k T}{6 \pi \eta R}
$$

where $k$ is the Boltzmann's constant, $T$ is temperature and $\eta$ is the viscosity of the medium. The nanoparticles were dispersed into $10 \mathrm{ml}$ of water and sonicated for $5 \mathrm{~min}$ before size measurement.

Furthermore, in the same instrument, the zeta potential method was used to measure the electrostatic potential of the $\mathrm{ZnO}-\mathrm{NP}$ surrounded by an aqueous medium, usually ultrapure water. Nanoparticles with a zeta potential between -10 and $+10 \mathrm{mV}$ are considered neutral. On the other hand, nanoparticles out of the range -30 to $30 \mathrm{mV}$ are considered strongly anionic and cationic, respectively [44].

\subsubsection{X-Ray Diffraction Spectroscopy (XRD)}

The synthesized ZnO-NP were structurally characterized by using a Rigaku MiniFlex/600 X-ray diffraction instrument, Japan. XRD operates at $30 \mathrm{kV}$ with a current of $15 \mathrm{~mA}$ using $\mathrm{Cu}-\mathrm{K} \alpha$ radiation (1.540598 $\AA$ wavelength) over a wide range of Bragg's angles (5 to $80,2 \theta$ degree). The average crystallite size $(L)$ of the $\mathrm{ZnO}-\mathrm{NP}$ was calculated by using the Debby Scherrer's equation and it is demonstrated as follows:

$L=\frac{K \lambda}{\phi \operatorname{Cos}(\theta)}$

where $K$ is the Scherrer constant (0.9), $\lambda$ is the X-ray radiation wavelength (1.540598 $\AA$ ), $\phi$ the full width half maximum height (FWHM) of the diffraction peak at the Bragg's angle $(\theta)$. relation:

The interplanar or reticular distance $(d)$, was calculated using Bragg's law given by the following

$d=\frac{n \lambda}{2 \operatorname{Sin}(\theta)}$

where $n$ is the Bragg order (a positive integer, 1) and $\lambda$ is the X-ray radiation wavelength (1.540598 $\AA$ ), and $\theta$ is the Bragg diffraction angle.

Average lattice parameters $(a, c)$, of the $\mathrm{ZnO}-\mathrm{NP}$ hexagonal crystal system, were calculated by using the equation:

$d(h k l)=\left[\frac{4}{3 a^{2}}\left(h^{2}+k^{2}+h k\right)+\frac{l^{2}}{c^{2}}\right]^{-1 / 2}$

where $h k l$ are the Miller indices of the reticular planes at the corresponding Bragg diffraction angles and $d$ is the reticular distance. 


\subsubsection{Attenuated Total Reflection-Fourier Transform Infrared Spectroscopy (ATR-FTIR)}

The FTIR measurement was carried out to identify the presence of characteristic bands of the $\mathrm{ZnO}$ products, and residues remaining before and after the thermal treatment. It was performed by using a Shimadzu IRTracer-100 spectrometer, Japan. Functional groups presence in the mid-infrared region (within the range of 400 to $4000 \mathrm{~cm}^{-1}$ ) were revealed in both samples, unannealed and annealed $\mathrm{ZnO}-\mathrm{NP}$. The samples were used in solid form. The Pike MIRacle single reflection horizontal ATR accessory equipped with a ZnSe ATR crystal was used for the analysis. ATR mode was used for recording the data.

\subsubsection{Field Emission Scanning Electron Microscopy (FE-SEM)}

Scanning electron microscopy analysis was used to determine the size and surface morphology of the synthesized ZnO-NP. FE-SEM characterization was carried out by using a FE-SEM, Hitachi Regulus 8230, Hitachi High-Tech Co., Japan. The instrument was operating at an accelerating voltage of $3.0 \mathrm{kV}$ and the obtained images were taken at a constant magnification of $40.0 \mathrm{kX}$. The sample studied did not require any preparation for measurement, as is often experimentally necessary.

\section{Results and Discussion}

\subsection{UV-Vis Analysis}

The UV-Vis spectrum (shown in Fig. 2) revealed a characteristic absorption peak of ZnO-NP at the wavelength of $361.5 \mathrm{~nm}$. It indicates that $\mathrm{ZnO}-\mathrm{NP}$ displays, at room temperature, an excitation absorption due to their large excitation binding energy. Furthermore, the absorption peak position confirms the blueshift spectra with concerning the bulk at $377 \mathrm{~nm}$ [45]. Quantum confinement of electrons in the semiconductor, due to the nanoscale, confirms the bandgap increase caused by ZnO-NP formation biosynthesized via leaf extract of Moringa oleifera.

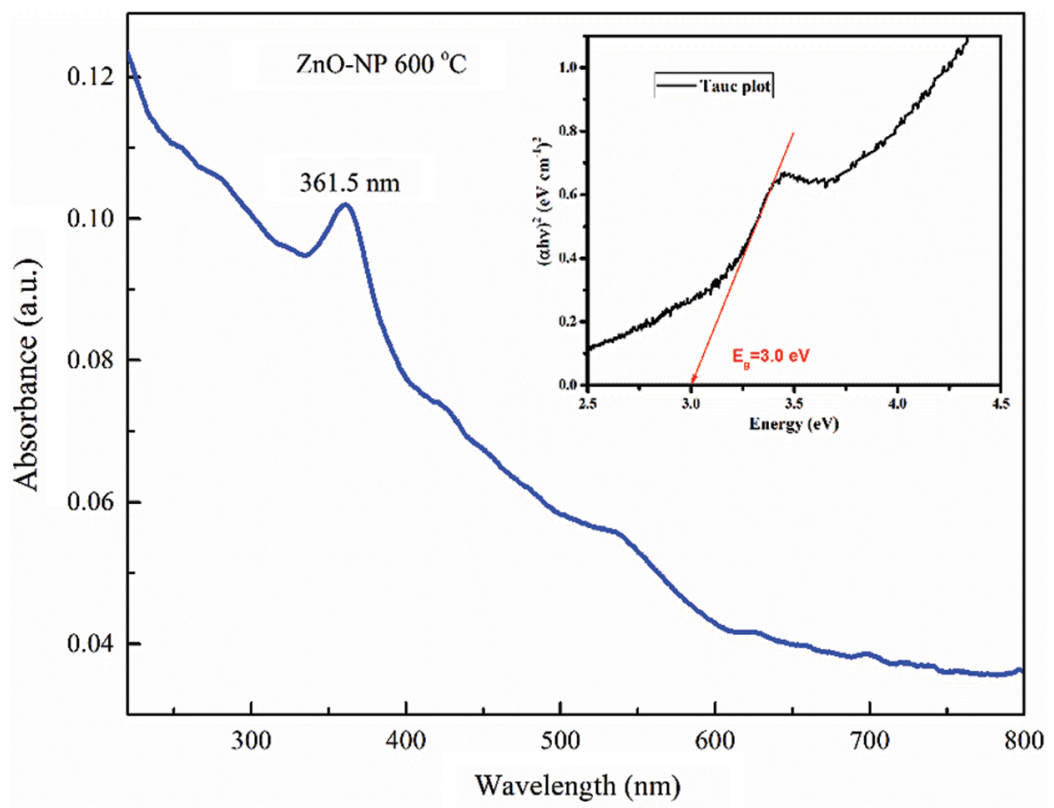

Figure 2: UV-Visible absorption spectroscopy of green synthesis $\mathrm{ZnO}-\mathrm{NP}$ annealed at temperature of $600^{\circ} \mathrm{C}$

To determine the optical bandgap of $\mathrm{ZnO}-\mathrm{NP}$, the optical absorption edge energy must be considered since it represents the minimum energy required to excite an electron from the highest occupied molecular orbital in the valence band to the lowest unoccupied molecular orbital in the conduction band [46]. It could be obtained by implementing the Tauc plot method based on Eqs. (1) and (2). In this case, 
the standard thickness of the cuvette used in UV-Vis instrument of $1 \mathrm{~cm}$ as $t$ value in Eq. (2) was considered. The $n$ value can take the following four values depending on the electronic transition in the $k$-space: $n=1 / 2$ for direct allowed transition, $n=2$ for indirect allowed transition, $n=3 / 2$ for direct forbidden transition and $n=3$ for indirect forbidden transition. As it is known, $\mathrm{ZnO}$ material has direct allowed transition $(n=1 / 2)$. For a practical representation, Eq. (1) could be rewritten as:

$$
(\alpha h v)^{2} \sim B\left(h v-E_{g}\right)
$$

This Tauc relation was plotted in Fig. 2 (inset). By simple observation, the intersection of the slope with the abscissa axis determines the bandgap of the material. In our case, an optical bandgap energy of $3.0 \mathrm{eV}$ for $\mathrm{ZnO}-\mathrm{NP}$ was found. It could be noted that this energy is lower than optical bandgap energy of $\mathrm{ZnO}$ bulk material $(\sim 3.3 \mathrm{eV})$. It could be associated with crystal defects, stress, or a nonstoichiometric phase in the nanoparticles caused by oxygen vacancies, for instance $[47,48]$.

In Tauc plot method, shown in Fig. 2 (inset), the Urbach tail region could be observed. This line shape region is very sensible to structural and chemical defects contributing in the determination of band-to-band transition by rising the tail. It usually causes large errors when bandgap energy is analyzed by Tauc method. Despite this, important information could be obtained about the synthesized $\mathrm{ZnO}-\mathrm{NP}$ sample, for example a high possibility of not getting a perfect crystal (or crystallite) via green synthesis by using Moringa oleifera leaves extract. This could be relevant when nanoparticles are used in technology, but not in agriculture as fertilizers, for instance.

\subsection{DLS and Zeta Potential Analysis}

The size distribution image obtained by DLS of green synthesized ZnO-NP is shown in Fig. 3. DLS analysis shows that the calculated average particle size distribution of these nanoparticles is $47.2 \mathrm{~nm}$ and the corresponding standard deviation is $3.2 \mathrm{~nm}$. Furthermore, particle size distribution by number indicates that $99.1 \%$ of the $\mathrm{ZnO}-\mathrm{NP}$ corresponds to the previously described nanoparticles. Several applications of nanoparticles need to guarantee a tight distribution of small nanoparticles because larger particles tend to agglomerate to a greater degree resulting in sedimentation. However, this phenomenon cannot be avoided, and the agglomerates are usually termed as secondary particles. Usually, particle diameter measured by DLS is larger than the ones obtained by other methods. ZnO-NP dispersed in ultrapure water do not keep their primary particle size because the attractive force between particles [43]. Also, the DLS technique obtains a hydrodynamic diameter based on the diffusion of the particles.

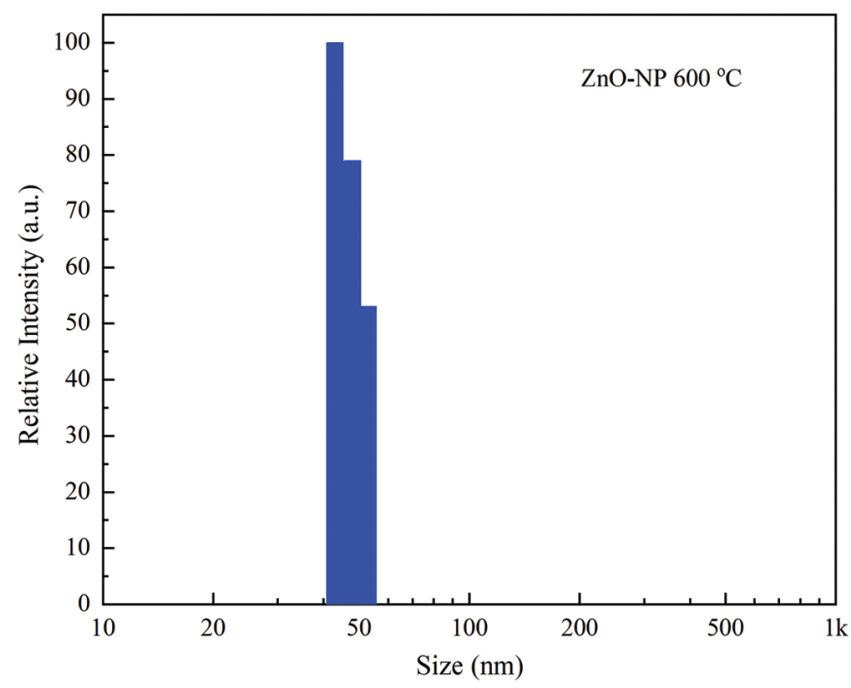

Figure 3: Dynamic light scattering particle size distribution by number-weighted of green synthesis $\mathrm{ZnO}-$ $\mathrm{NP}$ annealed at $600^{\circ} \mathrm{C}$ 
As mentioned previously, the magnitude of the zeta potential indicates potential stability of ZnO-NP in an aqueous medium. For the sample annealed at temperature of $600^{\circ} \mathrm{C}$, the zeta potential value was $-29.73 \mathrm{mV}$ (see Fig. 4), which indicates that the particles have a high stability, in this case ZnO-NP in ultrapure water.

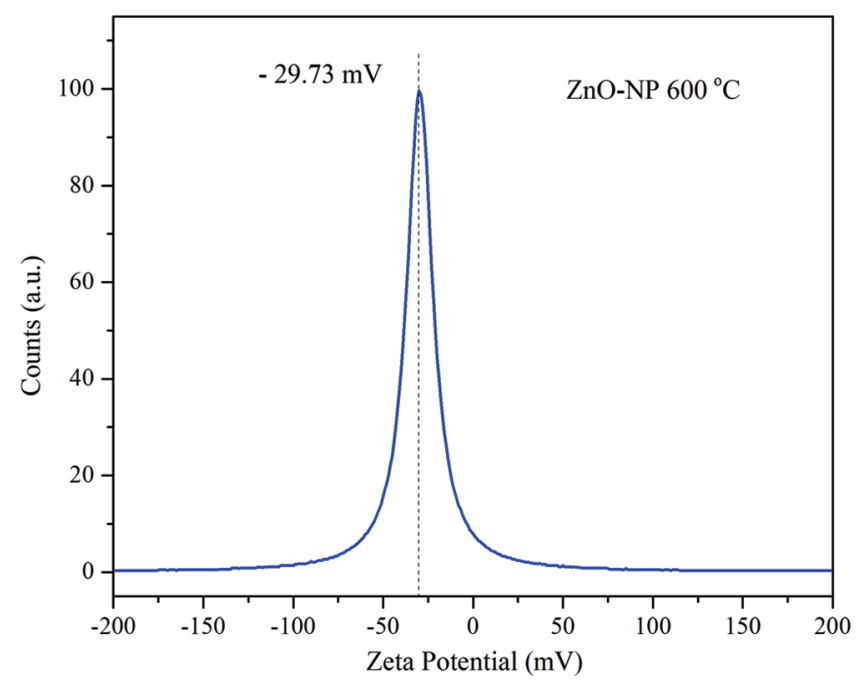

Figure 4: Zeta potential distribution of $\mathrm{ZnO}-\mathrm{NP}$ annealed at $600^{\circ} \mathrm{C}$

In a lot of cases, the $\mathrm{ZnO}-\mathrm{NP}$ have been used in an aqueous medium, resulting the zeta potential characterization of a primary indicator of surface charge. For example, in nanotoxicology ZnO-NP have been commonly utilized as a biological agent to control complex processes [49]. The initial nanoparticle size is important besides their final condition. This size nanoparticle stability is usually controlled by changing $\mathrm{pH}$ conditions to avoid agglomeration induced by solvation forces, van der Waals forces, electrostatic attractions, or/and hydrophobic interactions [50]. Zeta potential measurements bigger than $+/-30 \mathrm{mV}$ reveal suitable material avoiding $\mathrm{pH}$ control in solutions, for instance. ZnO-NP biosynthesized presented in this work exposes an appropriate nature to be applied in similar cases as the above mentioned.

\subsection{XRD Analysis}

The XRD patterns show the noticeable peaks of ZnO-NP indexed to (100), (002), (101), (102), (110), (103), (200), (112), and (201) planes of reflection of $\mathrm{ZnO}$ in agreement with Joint Committee on Powder Diffraction Standards (JCPDS) card number 01-079-0206 shown in Fig. 5a. All recorded peak intensity profiles, zoomed in the range of 25 to 70 degrees $(2 \theta)$, were characteristics of the hexagonal ZnO-NP wurtzite structure. Additionally, from the XRD pattern, no other diffraction peaks were observed, meaning there are not significant impurities in the $\mathrm{ZnO}$ crystallite.

Annealed sample of $\mathrm{ZnO}-\mathrm{NP}$ at the temperature of $600^{\circ} \mathrm{C}$ reveals well-defined, strong, and narrow diffraction peaks indicating a crystallite nature (see Fig. 5c). Furthermore, no additional peaks can be detected within the resolution limit of the instrument, in this way, the trend of a single-phase formation could be confirmed. However, the unannealed sample presents a convolution of the last three broadened peaks associated with (200), (112), and (201) planes of reflections (see Fig. 5b). A correlation between diffraction and lattice imperfectness is associated to size broadening independent of the diffraction order, anisotropies, and/or crystallite smallness [51]. 
JRM, 2022, vol.10, no.3

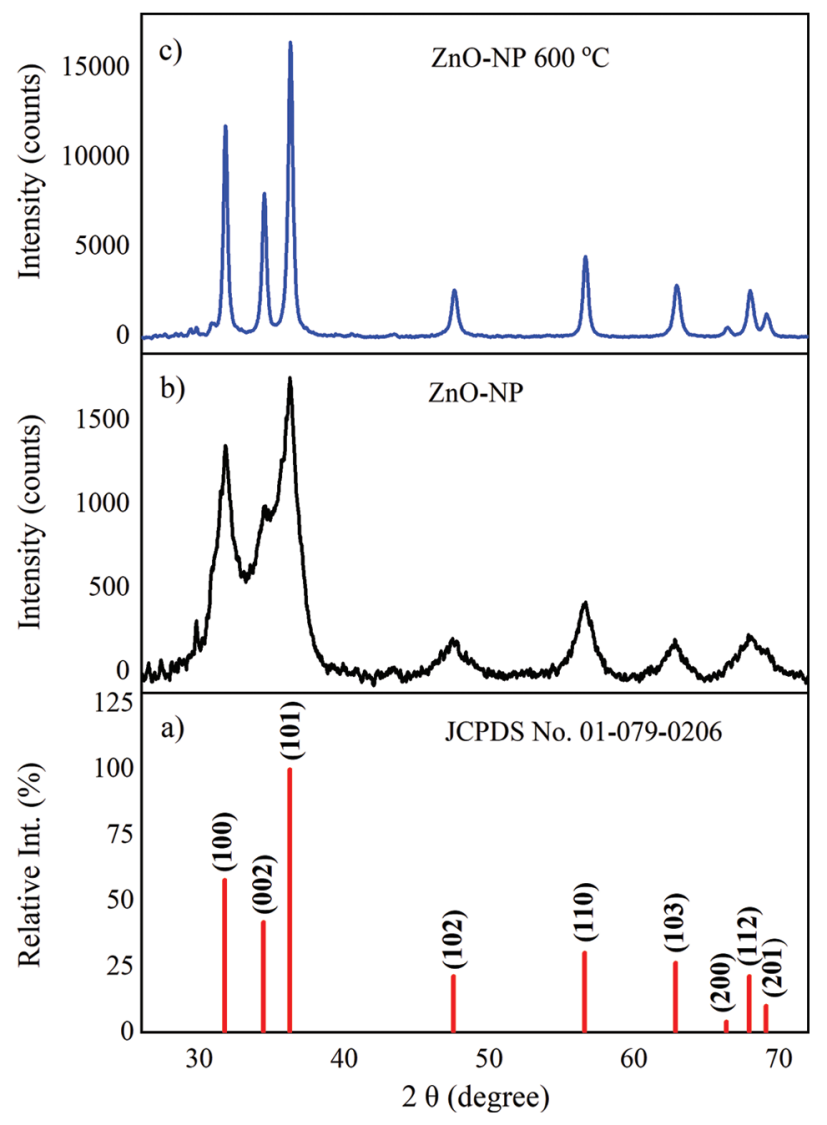

Figure 5: X-ray diffraction (a) relative intensity of $\mathrm{ZnO}$ reference card, and patterns of $\mathrm{ZnO}-\mathrm{NP}$ (b) unannealed and (c) annealed at temperature of $600^{\circ} \mathrm{C}$

By using the relation described in Eq. (6), the lattice parameters $(a, c)$, of the $\mathrm{ZnO}$ hexagonal structure are summarized in Tab. 1 as average values obtained from unannealed and annealed samples. These values have a good agreement with $\mathrm{ZnO}$ bulk parameters from the literature [52]. An insignificant variation of the lattice parameters ratio $(c / a)$ between unannealed (1.6039) and annealed (1.6006) concerning the bulk (1.6021) parameters are observed, as expected.

Table 1: Average lattice parameters of unannealed and annealed $\mathrm{ZnO}-\mathrm{NPs}\left(600^{\circ} \mathrm{C}\right)$

\begin{tabular}{lll}
\hline Material & $\langle a\rangle(\AA)$ & $\langle c\rangle(\AA)$ \\
\hline $\mathrm{ZnO}$ unannealed & 3.2491 & 5.2113 \\
$\mathrm{ZnO}$ annealed at $600^{\circ} \mathrm{C}$ & 3.2471 & 5.1972 \\
$\mathrm{ZnO}$ bulk & 3.2499 & 5.2066 \\
\hline
\end{tabular}

XRD technique was used not only for the identification of crystal structure but also for different lattice parameters. Interplanar distances for each Miller indices were calculated by using Brag's law, Eq. (5), associated with the Brag diffraction angle observed in Fig. 5. Summarized parameters as Miller index $(h k l)$, Brag diffraction angle $(\theta)$, interplanar distance $(d)$, and FWHM $(\phi)$, are shown in Tab. 2. 
Experimental position peaks have a good and clear correspondence to the $\mathrm{ZnO}-\mathrm{NP}$ reported in the literature. A minimal difference of the interplanar distance is observed between samples annealed $\left(d_{600 C}\right)$, unannealed $\left(d_{U A}\right)$, and bulk $\left(d_{\text {bulk }}\right)$ [52]. Furthermore, obtained values are in agreement with lattice parameters ratio $(c / a)$ above mentioned.

Table 2: Crystallinity parameters of bulk, unannealed and annealed at $600^{\circ} \mathrm{C}$ of $\mathrm{ZnO}-\mathrm{NPs}$

\begin{tabular}{lllllllll}
\hline$h k l$ & $2 \theta_{\text {bulk }}\left({ }^{\circ}\right)$ & $2 \theta_{U A}\left({ }^{\circ}\right)$ & $2 \theta_{600 C}\left({ }^{\circ}\right)$ & $d_{\text {bulk }}(\AA)$ & $d_{U A}(\AA)$ & $d_{600 C}(\AA)$ & $\phi_{U A}\left({ }^{\circ}\right)$ & $\phi_{600 C}\left({ }^{\circ}\right)$ \\
\hline$(100)$ & 31.768 & 31.7958 & 31.8063 & 2.8145 & 2.8121 & 2.8112 & 1.8843 & 0.3861 \\
$(002)$ & 34.422 & 34.3899 & 34.4862 & 2.6033 & 2.6057 & 2.5986 & 1.8260 & 0.4239 \\
$(101)$ & 36.253 & 36.2525 & 36.2938 & 2.4759 & 2.4760 & 2.4732 & 1.6421 & 0.4431 \\
$(102)$ & 47.539 & 47.5004 & 47.6070 & 1.9111 & 1.9126 & 1.9086 & 1.8329 & 0.5461 \\
$(110)$ & 56.594 & 56.5714 & 56.6503 & 1.6250 & 1.6256 & 1.6235 & 1.4774 & 0.4587 \\
$(103)$ & 62.858 & 62.7537 & 62.9563 & 1.4773 & 1.4795 & 1.4752 & 1.2708 & 0.5671 \\
\hline
\end{tabular}

FWHM is also reported in Tab. 2. It is known that the size of the particle increases while decreasing the values of FWHM. By using Debby Scherrer's formula, shown in Eq. (4), the crystallite average size of prepared $\mathrm{ZnO}-\mathrm{NP}$ was calculated 5.26 and $18.82 \mathrm{~nm}$ for unannealed and annealed samples, respectively. For annealed at the temperature of $600^{\circ} \mathrm{C}$, crystallite size obtained from XRD is smaller or equals to that of DLS and/or microscopy techniques (i.e., FE-SEM), as reported behavior in literature [43]. This fact can be easily revealed in this work by comparing XRD analysis, DLS analysis, and FE-SEM images.

On the other hand, analyzing the crystallographic results of the two types of samples, unannealed and annealed, there are slight reductions in parameters $a, c, d$ and consequently in the $c /$ a ratio after heat treatment, see Tabs. 1 and 2. These reductions suggest that the crystalline lattice suffered a slight contraction caused by the heat treatment applied on $\mathrm{ZnO}-\mathrm{NP}$.

\subsection{ATR-FTIR Analysis}

ATR-FTIR spectra of $\mathrm{ZnO}-\mathrm{NP}$ unannealed and annealed at $600^{\circ} \mathrm{C}$ in the spectral range of 400 to $4000 \mathrm{~cm}^{-1}$ are showed in Fig. 6. In both spectra, well-defined characteristic bands are evidenced at $\sim 1120$ and $\sim 416 \mathrm{~cm}^{-1}$ related to the deformation of the $\mathrm{Zn}-\mathrm{O}$ bond that indicates the presence of $\mathrm{ZnO}-\mathrm{NP}$ as a product of the synthesis carried out. Additionally, wide bands of different intensity related to the deformation of the $\mathrm{OH}$ bond are observed in $\sim 3405 \mathrm{~cm}^{-1}$ in the case of the product before heat treatment and $\sim 3506 \mathrm{~cm}^{-1}$ in the case of the synthesis product after heat treatment, this last deformation is much more subtle due to the removal of the organic fraction during the heat treatment. On the other hand, a transition is observed in the bands referring to the $\mathrm{C}=\mathrm{C}$ and $\mathrm{C}-\mathrm{C}$ bonds before and after the heat treatment, a transition that is due to the removal of the organic fraction, thus in the case of the sample without treatment, a series of bands between $\sim 1417-1604 \mathrm{~cm}^{-1}$ and an asymmetric band at $\sim 1417 \mathrm{~cm}^{-1}$ are visualized in the case of the spectrum of the post-treatment sample.

\subsection{FE-SEM Analysis}

A general arrangement of the annealed ZnO-NP sample obtained by FE-SEM is presented in Fig. 7a. In this overall micrograph, it is possible to observe a homogeneous dispersion and distribution in the absence of agglutination, agglomeration, or generation of significant lumps in the analyzed particles, enjoying simple contact with other surrounding nanoparticles. Likewise, there are also homogeneously distributed spaces or voids throughout the micrograph that suggests the stability concerning the dispersibility of this 
material in the dry state, this quality is essential to maintain its properties such as reactivity, electrostatic charge, qualities surface, among others, that must be considered for various applications. Different works associated with the development of nanoparticles of different kinds show in their morphological analyzes this type of agglomeration in different degrees at similar scales [53-55].

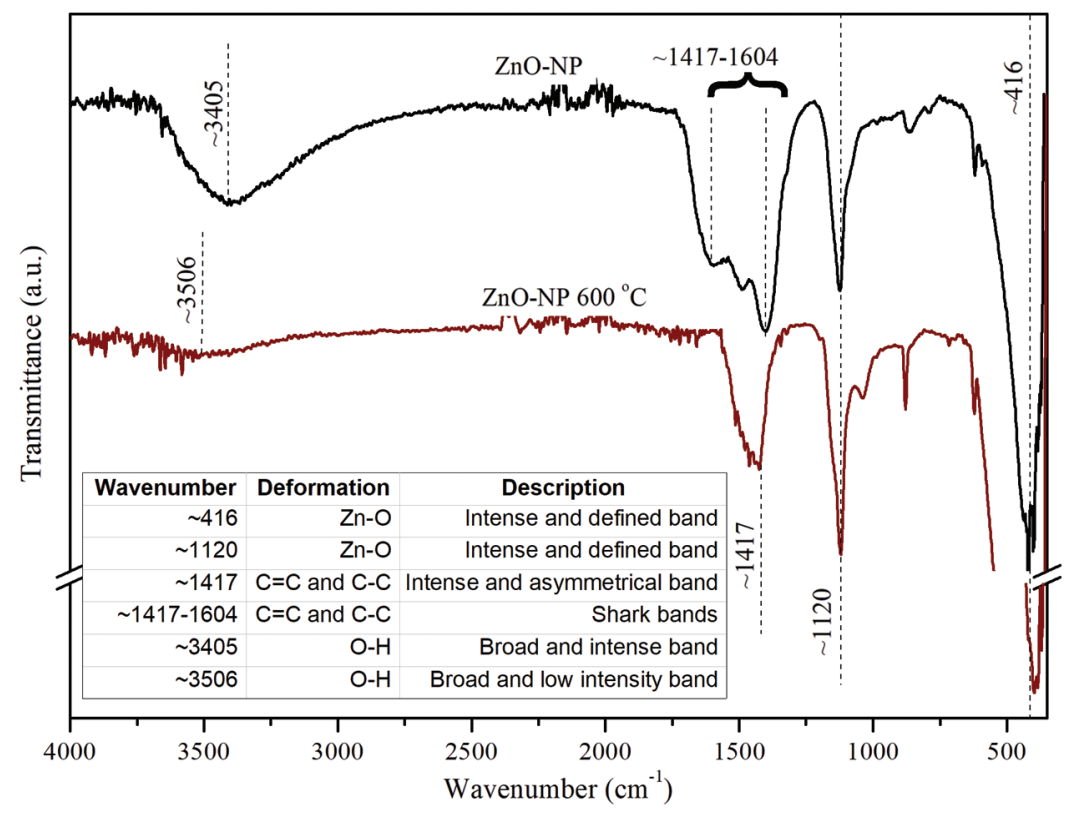

Figure 6: ATR-FTIR spectra of unannealed and annealed $\mathrm{ZnO}-\mathrm{NPs}$
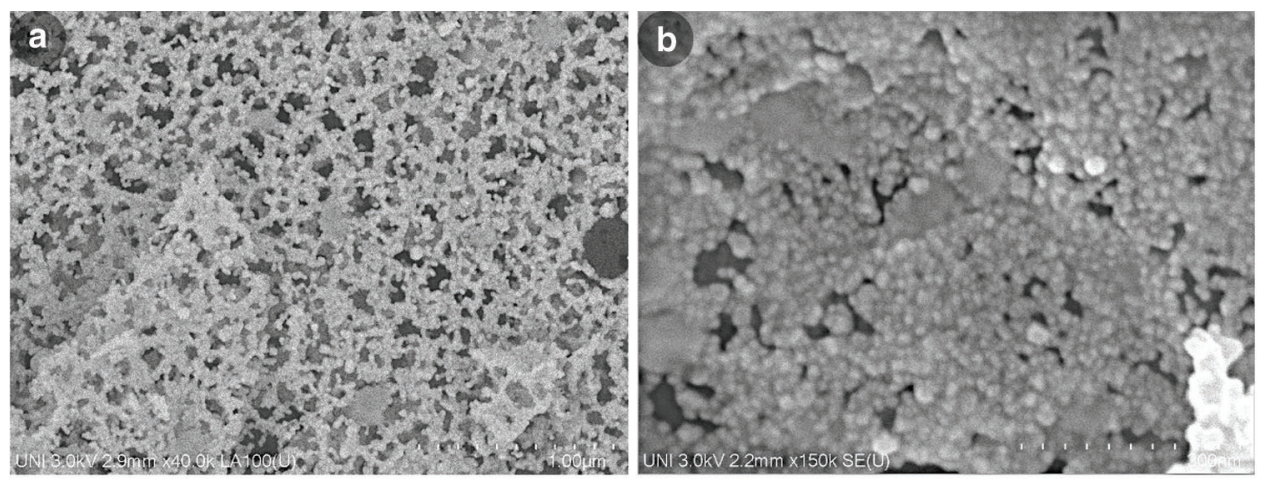

Figure 7: FE-SEM image of $\mathrm{ZnO}-\mathrm{NP}$ annealed at $600^{\circ} \mathrm{C}$ in the range of (a) $1 \mu \mathrm{m}$ and (b) $300 \mathrm{~nm}$

Although the sample is agglomerated due to the arrangement for taking the micrograph (see Fig. 7b), it is possible to distinguish particles with predominantly spheroidal morphologies, diameter of $25 \mathrm{~nm}$, and a granulometric distribution without great variations with sizes less than $47.2 \mathrm{~nm}$ as previously referred in DLS. In this context, and considering that the FE-SEM technique allows direct measurement of nanoparticles with reduced errors, it can be inferred that the FE-SEM, XRD, DLS, and, to a lesser extent, UV-Vis techniques have coherence in all their results. 


\section{Conclusions}

In this study, $\mathrm{ZnO}$ nanoparticles were successfully synthesized by using an eco-friendly green synthesis instead of conventional physical or chemical methods. Moringa oleifera leaves extract was utilized as a reductant and as a capping agent during synthesis. Morphological and structural properties of ZnO-NP were analyzed by UV-Vis, DLS, zeta potential, XRD, ATR-FTIR, and FE-SEM techniques. The data not only confirms the synthesis of ZnO-NP but also the absence of impurities associated with organic compounds in the annealed sample observed by ATR-FTIR and XRD spectroscopy. UV-Vis showed a peak absorbance at $361.5 \mathrm{~nm}$, indicating the presence of nanosized material in the aqueous medium, while DLS exposed a mean hydrodynamic diameter of $47.2 \mathrm{~nm}$ of the $\mathrm{ZnO}-\mathrm{NP}$. We found a zeta potential value of $-29.73 \mathrm{mV}$, which means that $\mathrm{NP}$ have a high stability in a water solution. By FE-SEM technique, a mean size diameter of $25 \mathrm{~nm}$ was obtained by direct measurement. XRD analysis yielded a similar diameter result $(18.82 \mathrm{~nm})$ for annealed $\mathrm{ZnO}-\mathrm{NP}$. It was determined that the heat annealing induced the crystallite size favoring monocrystallinity analyzed by XRD. Also, it was observed that the crystalline lattice suffered a slight contraction caused by the heat treatment applied on ZnO-NP. By doing a Tauc plot method, a bandgap energy of $3.0 \mathrm{eV}(\sim 300 \mathrm{meV}$ less than bulk) was obtained. In our case, this green synthesized material has a big influence on the Urbach region in the analysis, it means that the crystallite has defects, stress, or a nonstoichiometric phase in the nanoparticles probably caused by oxygen vacancies. In FE-SEM images a homogeneous dispersion and distribution in the absence of agglutination, agglomeration, or generation of significant lumps of the $\mathrm{ZnO}-\mathrm{NP}$ is observed. The experimental results let us conclude that the FE-SEM, XRD, DLS, and UV-Vis techniques have a dimensional coherence of synthesized ZnO-NP. Finally, owing to their optical and structural properties, and low-cost production, the synthesized ZnO-NP are proposed as useful materials in several applications as a follow-up of this study.

Acknowledgement: The authors are grateful to Dr. J. Quispe and Dr. H. Loro for their valuable support, to the Centro de Investigaciones Tecnológicas, Biomédicas y Medioambientales (CITBM) and finally to the Facultad de Ciencias of Universidad Nacional de Ingeniería.

Funding Statement: Authors are grateful to Concytec-Peru and The World Bank for the financial support of this project under the call "Mejoramiento y Ampliación de los Servicios del Sistema Nacional de Ciencia Tecnología e Innovación Tecnológica" 8682-PE, through Fondecyt Grant 017-2019 FONDECYT BM INC.INV.

Conflicts of Interest: The authors declare that they have no conflicts of interest to report regarding the present study.

\section{References}

1. Wu, J., Xue, D. (2011). Progress of science and technology of $\mathrm{ZnO}$ as advanced material. Science of Advanced Materials, 3(2), 127-149. DOI 10.1166/sam.2011.1144.

2. Vyas, S. (2020). A short review on properties and applications of zinc oxide based thin films and devices: $\mathrm{ZnO}$ as a promising material for applications in electronics, optoelectronics, biomedical and sensors. Johnson Matthey Technology Review, 64(2), 202-218. DOI 10.1595/205651320X15694993568524.

3. Yao, T. (2001). Zinc oxide. Encyclopedia of materials: Science and technology. Netherlands: Elsevier.

4. Singh, S. C. (2013). Zinc oxide nanostructures: Synthesis, characterizations and device applications. Journal of Nanoengineering and Nanomanufacturing, 3(4), 283-310. DOI 10.1166/jnan.2013.1147.

5. Vittal, R., Ho, K. C. (2017). Zinc oxide based dye-sensitized solar cells: A review. Renewable and Sustainable Energy Reviews, 70(52), 920-935. DOI 10.1016/j.rser.2016.11.273. 
JRM, 2022, vol.10, no.3

6. Sirelkhatim, A., Mahmud, S., Seeni, A., Kaus, N. H. M., Ann, L. C. et al. (2015). Review on zinc oxide nanoparticles: Antibacterial activity and toxicity mechanism. Nano-Micro Letters, 7(3), 219-242. DOI 10.1007/ s40820-015-0040-x.

7. Mirzaei, H., Darroudi, M. (2017). Zinc oxide nanoparticles: Biological synthesis and biomedical applications. Ceramics International, 43(1), 907-914. DOI 10.1016/j.ceramint.2016.10.051.

8. Hahn, Y. B. (2011). Zinc oxide nanostructures and their applications. Korean Journal of Chemical Engineering, 28(9), 1797-1813. DOI 10.1007/s11814-011-0213-3.

9. Ong, C. B., Ng, L. Y., Mohammad, A. W. (2018). A review of $\mathrm{ZnO}$ nanoparticles as solar photocatalysts: Synthesis, mechanisms and applications. Renewable and Sustainable Energy Reviews, 81(Suppl. 2), 536-551. DOI 10.1016/j.rser.2017.08.020.

10. Racca, L., Canta, M., Dumontel, B., Ancona, A., Limongi, T. et al. (2018). Zinc oxide nanostructures in biomedicine. Smart nanoparticles for biomedicine. Netherlands: Elsevier.

11. Kang, Y., Yu, F., Zhang, L., Wang, W., Chen, L. et al. (2021). Review of ZnO-based nanomaterials in gas sensors. Solid State Ionics, 360(7), 115544. DOI 10.1016/j.ssi.2020.115544.

12. Xu, X., Guo, H., Wang, X., Zhang, M., Wang, Z. et al. (2019). Physical properties and anti-aging characteristics of asphalt modified with nano-zinc oxide powder. Construction and Building Materials, 224, 732-742. DOI 10.1016/ j.conbuildmat.2019.07.097.

13. Luzi, F., Fortunati, E., Jiménez, A., Puglia, D., Chiralt, A. et al. (2017). PLA nanocomposites reinforced with cellulose nanocrystals from Posidonia oceanica and $\mathrm{ZnO}$ nanoparticles for packaging application. Journal of Renewable Materials, 5(2), 103-115. DOI 10.7569/JRM.2016.634135.

14. Borysiewicz, M. A. (2019). $\mathrm{ZnO}$ as a functional material, a review. Crystals, 9(10), 505. DOI 10.3390/ cryst9100505.

15. Agarwal, H., Shanmugam, V. K. (2020). A review on anti-inflammatory activity of green synthesized zinc oxide nanoparticle: Mechanism-based approach. Bioorganic Chemistry, 94, 103423. DOI 10.1016/j. bioorg.2019.103423.

16. Zhu, X., Pathakoti, K., Hwang, H. M. (2019). Green synthesis of titanium dioxide and zinc oxide nanoparticles and their usage for antimicrobial applications and environmental remediation. Green synthesis, characterization and applications of nanoparticles. Netherlands: Elsevier.

17. Theerthagiri, J., Salla, S., Senthil, R. A., Nithyadharseni, P., Madankumar, A. et al. (2019). A review on ZnO nanostructured materials: Energy, environmental and biological applications. Nanotechnology, 30(39), 392001. DOI 10.1088/1361-6528/ab268a.

18. Sabir, S., Arshad, M., Chaudhari, S. K. (2014). Zinc oxide nanoparticles for revolutionizing agriculture: Synthesis and applications. The Scientific World Journal, 2014(3), 1-8. DOI 10.1155/2014/925494.

19. Zarazua-Aguilar, Y., Paredes-Carrera, S. P., Sánchez-Ochoa, J. C., Avendano-Gómez, J. R., Flores-Valle, S. O. (2017). Influence of microwave/ultrasound irradiation on the sol-gel synthesis of titanium dioxide nanoparticles for application in photocatalysis. Revista Mexicana De Ingenieria Quimica, 16(3), 899-909. http://rmiq.org/ ojs311/index.php/rmiq/article/view/977.

20. Daruich de Souza, C., Ribeiro Nogueira, B., Rostelato, M. E. C. M. (2019). Review of the methodologies used in the synthesis gold nanoparticles by chemical reduction. Journal of Alloys and Compounds, 798(47), 714-740. DOI 10.1016/j.jallcom.2019.05.153.

21. Joshi, R. (2018). Facile photochemical synthesis of $\mathrm{ZnO}$ nanoparticles in aqueous solution without capping agents. Materialia, 2(2), 104-110. DOI 10.1016/j.mtla.2018.07.001.

22. El Filali, B., Torchynska, T. V., Díaz Cano, A. I., Morales Rodríguez, M. (2015). Structural and Raman scattering studies of $\mathrm{ZnO} \mathrm{Cu}$ nanocrystals grown by spray pyrolysis. Revista Mexicana De Ingeniería Química, 14(3), 781788. http://www.rmiq.org/ojs311/index.php/rmiq/article/view/1433.

23. Amendola, V., Meneghetti, M. (2009). Laser ablation synthesis in solution and size manipulation of noble metal nanoparticles. Physical Chemistry Chemical Physics, 11(20), 3805-3821. DOI 10.1039/b900654k.

24. Othman, A. A., Osman, M. A., Abd-Elrahim, A. G. (2018). The effect of milling time on structural, optical and photoluminescence properties of ZnO nanocrystals. Optik, 156(20), 161-168. DOI 10.1016/j.ijleo.2017.11.037. 
25. Córdova-Cisneros, K. C., Sáenz-Galindo, A., Ascacio-Valdés, J. A., Narro-Céspedes, R. I., Castañeda-Facio, A. (2021). Green synthesis of silver nanoparticles using the aqueous extract of larrea tridentate and eucalyptus. Revista Mexicana De Ingeniería Química, 20(1), 13-24. DOI 10.24275/rmiq/Mat1117.

26. Mohamed, A. A., Abu-Elghait, M., Ahmed, N. E., Salem, S. S. (2020). Eco-friendly mycogenic synthesis of ZnO and $\mathrm{CuO}$ nanoparticles for in vitro antibacterial, antibiofilm, and antifungal applications. Biological Trace Element Research, 199, 1-12. DOI 10.1007/s12011-020-02369-4.

27. Katiyar, A., Kumar, N., Shukla, R. K., Srivastava, A. (2020). Influence of alkali hydroxides on synthesis, physicochemical and photoluminescence properties of zinc oxide nanoparticles. Materials Today: Proceedings, 29(3), 885-889. DOI 10.1016/j.matpr.2020.05.112.

28. DeRosa, M., Monreal, C., Schnitzer, M., Walsh, R., Sultan, Y. (2010). Nanotechnology in fertilizers. Nature Nanotech, 5(2), 91-91. DOI 10.1038/nnano.2010.2.

29. Xu, C., de, S., Balu, A. M., Ojeda, M., Luque, R. (2015). Mechanochemical synthesis of advanced nanomaterials for catalytic applications. Chemical Communications, 51(31), 6698-6713. DOI 10.1039/C4CC09876E.

30. Otis, G., Ejgenberg, M., Mastai, Y. (2021). Solvent-free mechanochemical synthesis of ZnO nanoparticles by highenergy ball milling of $\varepsilon-\mathrm{Zn}(\mathrm{OH})_{2}$ crystals. Nanomaterials, 11(1), 238. DOI 10.3390/nano11010238.

31. Iravani, S., Korbekandi, H., Mirmohammadi, S. V., Zolfaghari, B. (2014). Synthesis of silver nanoparticles: Chemical, physical and biological methods. Research in Pharmaceutical Sciences, 9(6), 385-406. https://www. ncbi.nlm.nih.gov/pme/articles/PMC4326978/.

32. Naveed Ul Haq, A., Nadhman, A., Ullah, I., Mustafa, G., Yasinzai, M. et al. (2017). Synthesis approaches of zinc oxide nanoparticles: The dilemma of ecotoxicity. Journal of Nanomaterials, 2017(12), 1-14. DOI 10.1155/2017/ 8510342.

33. Kumar, B., Smita, K., Cumbal, L., Debut, A. (2014). Green approach for fabrication and applications of zinc oxide nanoparticles. Bioinorganic Chemistry and Applications, 2014(3), 1-7. DOI 10.1155/2014/523869.

34. Rana, A., Yadav, K., Jagadevan, S. (2020). A comprehensive review on green synthesis of nature-inspired metal nanoparticles: Mechanism, application and toxicity. Journal of Cleaner Production, 272(5), 122880. DOI 10.1016/ j.jclepro.2020.122880.

35. El Shafey, A. M. (2020). Green synthesis of metal and metal oxide nanoparticles from plant leaf extracts and their applications: A review. Green Processing and Synthesis, 9(1), 304-339. DOI 10.1515/gps-2020-0031.

36. Manguro, L. O. A., Lemmen, P. (2007). Phenolics of Moringa oleifera leaves. Natural Product Research, 21(1), 56-68. DOI 10.1080/14786410601035811.

37. Ngom, I., Ndiayea, N. M., Bakayoko, A. F. M., Ngom, B. D., Maaza, M. (2020). On the use of Moringa oleifera leaves extract for the biosynthesis of $\mathrm{NiO}$ and $\mathrm{ZnO}$ nanoparticles. MRS Advances, 5(21), 1145-1155. DOI 10.1557/adv.2020.212.

38. Iravani, S. (2011). Green synthesis of metal nanoparticles using plants. Green Chemistry, 13(10), 2638-2650. DOI $10.1039 / \mathrm{c} 1 \mathrm{gc} 15386 \mathrm{~b}$.

39. Aboulthana, W. M., Youssef, A. M., Seif, M. M., Osman, N. M., Sahu, R. K. et al. (2021). Comparative study between Croton tiglium seeds and Moringa oleifera leaves extracts, after incorporating silver nanoparticles, on murine brains. Egyptian Journal of Chemistry, 64(4), 1709-1731. DOI 10.21608/EJCHEM.2021.53777.3113.

40. Bandeira, M., Giovanela, M., Roesch-Ely, M., Devine, D. M., da Silva Crespo, J. (2020). Green synthesis of zinc oxide nanoparticles: A review of the synthesis methodology and mechanism of formation. Sustainable Chemistry and Pharmacy, 15, 100223. DOI 10.1016/j.scp.2020.100223.

41. Makarov, V. V., Love, A. J., Sinitsyna, O. V., Makarova, S. S., Yaminsky, I. V. et al. (2014). Green nanotechnologies: Synthesis of metal nanoparticles using plants. Acta Naturae, 6(1), 20-44. DOI 10.32607/ 20758251-2014-6-1-35-44.

42. Tauc, J., Grigorovici, R., Vancu, A. (1966). Optical properties and electronic structure of amorphous germanium. Physica Status Solidi (B), 15(2), 627-637. DOI 10.1002/(ISSN)1521-3951.

43. Akbari, B., Pirhadi Tavandashti, M., Zandrahimi, M. (2011). Particle size characterization of nanoparticles-a practical approach. Iranian Journal of Materials Science and Engineering, 8(2), 48-56. http://ijmse.iust.ac.ir/ article-1-341-en.html. 
44. Clogston, J. D., Patri, A. K. (2011). Zeta potential measurement. Characterization of nanoparticles intended for drug delivery. USA: Humana Press.

45. Srikant, V., Clarke, D. R. (1998). On the optical band gap of zinc oxide. Journal of Applied Physics, 83(10), $5447-$ 5451. DOI 10.1063/1.367375.

46. Sharma, N., Prabakar, K., Ilango, S., Dash, S., Tyagi, A. K. (2017). Optical band-gap and associated Urbach energy tails in defected AlN thin films grown by ion beam sputter deposition: Effect of assisted ion energy. Advanced Materials \& Processes, 2(5), 342-346. DOI 10.5185/amp.2017/511.

47. Bindu, P., Thomas, S. (2017). Optical properties of $\mathrm{ZnO}$ nanoparticles synthesised from a polysaccharide and $\mathrm{ZnCl}_{2}$. Acta Physica Polonica A, 131(6), 1474-1478. DOI 10.12693/APhysPolA.131.1474.

48. Caglar, M., Ilican, S., Caglar, Y. (2009). Influence of dopant concentration on the optical properties of ZnO: In films by sol-gel method. Thin Solid Films, 517(17), 5023-5028. DOI 10.1016/j.tsf.2009.03.037.

49. Berg, J. M., Romoser, A., Banerjee, N., Zebda, R., Sayes, C. M. (2009). The relationship between pH and zeta potential of $\sim 30 \mathrm{~nm}$ metal oxide nanoparticle suspensions relevant toin vitrotoxicological evaluations. Nanotoxicology, 3(4), 276-283. DOI 10.3109/17435390903276941.

50. Min, Y., Akbulut, M., Kristiansen, K., Golan, Y., Israelachvili, J. (2008). The role of interparticle and external forces in nanoparticle assembly. Nature Materials, 7, 527-538. DOI 10.1038/nmat2206.

51. Ungár, T. (2004). Microstructural parameters from X-ray diffraction peak broadening. Scripta Materialia, 51(8), 777-781. DOI 10.1016/j.scriptamat.2004.05.007.

52. Albertsson, J., Abrahams, S. C., Kvick, A. (1989). Atomic displacement, anharmonic thermal vibration, expansivity and pyroelectric coefficient thermal dependences in ZnO. Acta Crystallographica Section B, 45(1), 34-40. DOI 10.1107/S0108768188010109.

53. Al-Bedairy, M. A., Habeeb Alshamsi, H. A. (2018). Environmentally friendly preparation of zinc oxide, study catalytic performance of photodegradation by sunlight for rhodamine B dye. Eurasian Journal of Analytical Chemistry, 13(6), 1-9. DOI 10.29333/ejac/101785.

54. Khezli, S., Abedini, A. (2017). Effect of amino acids as a capping agent on the size and morphology of pure AgO nanoparticles and its photocatalyst application. Journal of Materials Science: Materials in Electronics, 28(14), 10535-10540. DOI 10.1007/s10854-017-6827-4.

55. Abed, H. H., Alwasiti, E. A., Tawfeeq, A. T. (2020). Titanium nanoparticles conjugated with streptokinase as a modified thrombolytic agent. International Journal of Current Pharmaceutical Research, 12(1), 18-25. DOI 10.22159/ijcpr.2020v12i1.36824. 